\title{
Prácticas no-epistémicas: ampliando la mirada en el enfoque didáctico basado en prácticas científicas
}

\author{
Antonio García-Carmona \\ Departamento de Didáctica de las Ciencias Experimentales y Sociales. Universidad de Sevilla. España. \\ garcia-carmona@us.es
}

[Recibido: 14 mayo 2020. Revisado: 8 julio 2020. Aceptado: 1 septiembre 2020]

Resumen: El presente artículo reivindica prestar una atención explícita a las prácticas no-epistémicas como ampliación de aquellos planteamientos predominantes del enfoque didáctico basado en prácticas científicas, que solo suelen estar focalizados en prácticas epistémicas. Para ello, (1) se exponen los principales argumentos empleados en promover este enfoque didáctico; (2) se analizan críticamente diferentes conceptualizaciones sobre el aprendizaje de la ciencia mediante prácticas científicas; (3) se justifica la integración de la dimensión noepistémica en tal enfoque a partir de bibliografía sobre filosofía, sociología e historia de la ciencia; y finalmente (4) se proponen de manera fundamentada algunas prácticas no-epistémicas que podrían abordarse en la educación científica básica.

Palabras clave: enseñanza de la ciencia; indagación; prácticas científicas; prácticas epistémicas; prácticas no-epistémicas.

Non-epistemic practices: extending the view in the didactic approach based on scientific practices

Abstract: This article calls for explicit attention to non-epistemic practices as an extension of the predominant approaches on practice-based to science education, which tend to emphasize mainly epistemic practices. To this end, (1) the main arguments used to promote this didactic approach are outlined; (2) different conceptualizations of science learning through scientific practices are critically analyzed; (3) the integration of the non-epistemic dimension in such an approach is justified on the basis of the literature on philosophy, sociology and history of science; and finally (4) the rationale for some non-epistemic practices that could be addressed in basic science education is proposed.

Keywords: epistemic practices; non-epistemic practices; inquiry; science education; scientific practices.

Para citar este artículo: García-Carmona, A. (2021) Prácticas no-epistémicas: ampliando la mirada en el enfoque didáctico basado en prácticas científicas. Revista Eureka sobre Enseñanza y Divulgación de las Ciencias 18(1), 1108. doi: 10.25267/Rev_Eureka_ensen_divulg_cienc.2021.v18.11.1108

La ciencia (...) es una actividad muy bumana, llena de sesgos y accidentes, conducida por egos y financiación, competitiva y a veces inmoral, fuertemente enmarcada en las agendas de las grandes instituciones y movimientos sociales: una comedia humana hermosa y terrible, como cualquier otra parte de la vida. (Lemke 1998; traducción propia)

\section{Introducción}

En los últimos años ha surgido una corriente didáctica que sostiene que una buena forma de aprender ciencia es mediante la participación en prácticas científicas (Ford 2015; Jiménez 2012; Kelly 2008; NRC 2012; Osborne 2014). A grandes rasgos, este planteamiento propone que, para aprender ciencia haciendo ciencia, se deben armonizar procesos cognitivos, habilidades procedimentales, así como conocimientos científicos y metacientíficos ${ }^{1}$ (Duschl 2008; GarcíaCarmona y Acevedo-Díaz 2018; Stroupe 2014, 2015). Según Bybee (2011), el enfoque basado en prácticas científicas no intenta reemplazar al modelo de aprendizaje por indagación, sino expandir y enriquecer la enseñanza/aprendizaje de la ciencia. De modo que, en este nuevo

\footnotetext{
${ }^{1}$ Esto es, una comprensión básica sobre la naturaleza del conocimiento y la actividad científica, a partir de
} aportaciones de la filosofía, la historia y la sociología de la ciencia.

\author{
Revista Eureka sobre Enseñanza y Divulgación de las Ciencias \\ Universidad de Cádiz. APAC-Eureka. ISSN: 1697-011X \\ bttp://doi.org/10.25267/Rev_Eureka_ensen_divulg_cienc.2021.v18.i1.1108 \\ http:/ / reuredcuca.es
}


marco, la indagación es considerada una práctica científica más entre otras (Bybee 2011; Michaels, Shouse y Schweingruber 2008).

Si bien, como sucede con otros planteamientos didácticos, para el aprendizaje de la ciencia mediante prácticas científicas se encuentran conceptualizaciones o perspectivas predominantes, que son susceptibles de ser analizadas y replanteadas desde otros puntos de vista. Por ejemplo, las propuestas más populares o seguidas en la bibliografía sobre este enfoque (e.g., NRC 2012) se ocupan, sobre todo, de la dimensión epistémica (i.e., racional o cognitiva) de la actividad científica (García-Carmona 2020). Sin embargo, a nuestro parecer, el enfoque sería más completo y coherente con el trabajo que realizan las científicas y científicos si se atienden, de igual modo, las múltiples prácticas de naturaleza no-epistémica que estos también desempeñan.

Así pues, para defender nuestra posición sobre el aprendizaje de la ciencia basado en prácticas científicas, presentamos este artículo con los siguientes objetivos:

1. Exponer los principales argumentos que han motivado el marco de aprendizaje de la ciencia mediante prácticas científicas;

2. analizar críticamente las conceptualizaciones más destacadas de la bibliografía sobre el enfoque basado en prácticas científicas;

3. justificar la integración de la dimensión no-epistémica en este enfoque con apoyo en bibliografía sobre filosofía, sociología e historia de la ciencia; y

4. proponer razonadamente algunas prácticas científicas no-epistémicas que podrían abordarse en la educación científica básica.

\section{¿Por qué una enseñanza de la ciencia basada en prácticas cientificas?}

En el panorama internacional de la enseñanza de la ciencia, la indagación está considerada como una de las formas más propicias de aprender ciencia (Abd-El-Khalick et al. 2004; OECD 2019; Harlen, 2013; Rocard et al. 2007). El origen de este enfoque didáctico se sitúa en los inicios del siglo XX con el estadounidense J. Dewey (1859-1952) como exponente pionero; aunque no fue hasta la década de 1960 cuando empieza a tener auge en las propuestas para la enseñanza de la ciencia en Estados Unidos (Barrow 2006).

En España, el enfoque basado en la indagación (en adelante, EBI) comenzó a tener cierto impacto a partir de la década de 1980 con propuestas que hacían referencia a enseñar y aprender ciencia como investigación escolar (Cañal y Porlán 1987) o mediante la resolución de problemas como investigación (Gil, Dumas, Maillot, Martínez-Torregrosa y Ramírez 1988), entre otras denominaciones parecidas. En la actualidad, la investigación didáctica que se desarrolla en nuestro país, al respecto, ha dejado de emplear esa terminología primigenia y ya solo se suele hablar de indagación ${ }^{2}$ (Aguilera et al. 2018).

Desde su génesis, el EBI ha sido interpretado de diversas maneras (Abell, Smith y Volkmann 2006; Cañal 1999; Garritz 2012). Si bien, en términos generales se puede decir que aprender ciencia en el marco del EBI implica que los estudiantes ( $\imath$ desarrollen ideas científicas mediante destrezas de cómo investigar y construir su propio conocimiento para entender y participar con responsabilidad en el mundo [físico] donde viven; y (ii) utilicen, para ello, procesos similares a los empleados por las personas dedicadas a la ciencia, tales como el planteamiento de preguntas, la recopilación de datos, el razonamiento y la revisión de pruebas a partir de lo

\footnotetext{
${ }^{2}$ Indagación es la traducción de inquiry que se ha impuesto en España, dentro de la didáctica de la ciencia. Según el Diccionario de la Real Academia Española (DRAE), indagar significa "Intentar averiguar algo discurriendo o con preguntas", y se puede considerar un sinónimo de investigar.
} 
que se sabe, la discusión de resultados y la obtención de conclusiones (Harlen 2012; InterAcademy Partnership 2010; NRC 1996).

Existe abundante bibliografía sobre la eficacia didáctica del aprendizaje de la ciencia mediante el EBI. ${ }^{3}$ Entre otros aspectos, favorece que los estudiantes activen el pensamiento, habilidades procedimentales y actitudes positivas hacia la ciencia (Anderson 2002; Minner, Levy y Century 2010). Sin embargo, se encuentran también dificultades en los estudiantes para elaborar argumentos y explicaciones científicas a partir de pruebas y razonamiento (Rönnebeck et al. 2016). Asimismo, el profesorado de ciencia manifiesta dificultades para asimilar e implementar el enfoque indagador en su práctica docente (Crawford 2007; Kim y Tan 2011; Yoon, Joung y Kim 2012).

Entre los posibles motivos de que el EBI no resulte todo lo eficaz que se espera para aprender ciencia, Osborne (2014) aduce una malinterpretación de este. Esto se pone de manifiesto, por ejemplo, con el hecho de que el EBI se haya traducido, a menudo, en la realización de simples experiencias de laboratorio, que los estudiantes llevan a cabo de manera acrítica y mecánica (Hodson 2005). A ello habría que añadir la multiplicidad de significados existente en la bibliografía con relación al término inquiry. Minner et al. (2010) encontraron que este suele emplearse para indicar: (i) lo que hacen las personas dedicadas a la ciencia (metodología científica), (ii) lo que hace el alumnado que aprende ciencia con estrategias inspiradas en la actividad científica (proceso de aprendizaje), y (iii) lo que hace el profesorado para implementar el currículo de ciencia escolar como indagación (método de enseñanza). Probablemente, esta variedad de significados ha podido obstaculizar también una conceptualización apropiada del EBI.

Asimismo, Osborne (2014) considera que algunas interpretaciones del EBI han llevado a equiparar hacer ciencia con aprender ciencia, pese a que son dos actividades distintas en finalidad y desarrollo. Michaels et al. (2008) tienen una percepción similar y, en lugar de aprendizaje como indagación, prefieren hablar de aprendizaje basado en practicas cientificas. Estos autores argumentan que "la ciencia como práctica implica hacer algo y aprender algo, de manera que "bacer" y "aprender" no se pueden separar realmente" (Michaels et al. 2008, p. 34; traducción propia). Por tanto, para estos autores las "practicas científicas" constituyen un enfoque didáctico más amplio que el EBI. Un análisis comparativo más profundo de ambos enfoques didácticos puede consultarse en García-Carmona (2020).

\section{¿Qué implica aprender ciencia mediante prácticas científicas?}

A partir de una revisión particular y no sistemática de bibliografía sobre filosofía de la ciencia y psicología, Osborne (2014) concluye que participar en prácticas científicas escolares solo tendrá sentido si: (a) ayuda a los estudiantes a desarrollar una comprensión amplia y profunda de lo que sabemos, de cómo lo sabemos y de los constructos que guían la práctica de la ciencia; $(b)$ constituye un medio más efectivo para desarrollar ese conocimiento; y $(c)$ presenta una imagen más auténtica de la ciencia.

En una línea similar, Ford (2015) argumenta que participar en practicas científicas no significa seguir una serie de reglas, sino más bien adquirir una capacidad para la evaluación y crítica continuas en la construcción de conocimiento científico. En tal sentido, Ford entiende que aprender ciencia mediante prácticas científicas: $(i)$ requiere de unos conocimientos específicos para participar en las distintas prácticas, que son distintos de las habilidades procedimentales generales; (ii) implica que las distintas prácticas no son independientes, sino que están necesariamente relacionadas; y (iii) enfatiza la conexión entre hacer y aprender.

\footnotetext{
${ }^{3}$ Véanse, por ejemplo, las revisiones recientes de Romero-Ariza (2017), Rönnebeck et al. (2016) y Zhang (2016), con una mirada internacional, y de Aguilera et al. (2018), para el contexto nacional.
} 
Kelly (2008) subraya el papel de la interacción social en el aprendizaje de la ciencia mediante prácticas científicas y, para ello, habla de prácticas epistémicas, que define como "las formas socialmente organizadas e intencionadamente establecidas, que emplean los miembros de una comunidad para proponer, comunicar, evaluar y legitimar el conocimiento dentro de un marco disciplinar." (p. 99; traducción propia). Así, Kelly y colegas consideran que las prácticas se aprenden mediante la participación e interacción con otros miembros de la misma comunidad de aprendizaje (Kelly y Licona 2018). Con lo cual, el conocimiento es concebido como un producto negociado entre las personas de un grupo social, a partir de discusiones basadas en razonamiento, persuasión, uso de datos, etc. (Kelly, McDonald y Wickman 2012). ${ }^{4}$

Jiménez-Aleixandre y Crujeiras (2017) se alinean con las perspectivas que se acaban de exponer en sus planteamientos sobre las prácticas científicas. No obstante, reconocen que los términos "prácticas científicas" y "prácticas epistémicas" se usan a menudo de manera indiferenciada en la bibliografía, aunque, sensu stricto, no significan lo mismo. Aun así, sugieren que, en el ámbito educativo, las prácticas científicas sean entendidas con un significado equivalente al de prácticas epistémicas (Jiménez-Aleixandre y Crujeiras 2017, p. 70). En un trabajo anterior, la primera autora lo expresa de la siguiente manera:

\begin{abstract}
Para la caracterización de estas prácticas seguimos a Kelly (2008), quien las denomina prácticas epistémicas. (...) utilizaremos el término prácticas científicas con un significado equivalente al de prácticas epistémicas de la ciencia, que resulta, creemos, de significado más claro en nuestro contexto. (Jiménez 2012, p. 11).
\end{abstract}

Los planteamientos anteriores se han visto reflejados en algunas propuestas específicas como, por ejemplo, la sugerida en el último documento de reforma para la educación científica preuniversitaria en Estados Unidos: “A Framework for K-12 Science Education” (NRC 2012). En la tabla 1 se recopilan las ocho prácticas de ciencia ${ }^{5}$ que propone tal documento. Se observa que las prácticas científicas seleccionadas por el NRC (2012) están sesgadas hacia una visión esencialmente epistémica de la ciencia; o, al menos, las sugerencias que se ofrecen en el documento para su desarrollo en el aula se enmarcan primordialmente en tal visión (GarcíaCarmona 2020). Lo cual, a nuestro modo de ver, proyecta una perspectiva restringida de las prácticas científicas de cara a su abordaje en la enseñanza/aprendizaje de la ciencia; puesto que (i) la actividad científica conlleva también la participación en prácticas de naturaleza noepistémica que, además, (ii) pueden ser analizadas y comprendidas por estudiantes de niveles educativos básicos (Acevedo-Díaz, García-Carmona y Aragón 2017).

Obviamente, se puede pensar que la selección y los planteamientos de las prácticas científicas recogidos en el documento "K-12 Framework" (NRC 2012) conforman tan solo una propuesta entre otras posibles que, además, está dirigida al profesorado de ciencia de un contexto educativo particular. Sin embargo, las recomendaciones de este documento son muy influyentes tanto en Estados Unidos como en otros muchos países. Resulta difícil encontrar, en la bibliografía internacional, estudios y experiencias sobre aprendizaje de la ciencia mediante prácticas científicas que no lo citen entre sus referencias básicas (García-Carmona 2020).

En el contexto español, por ejemplo, la propuesta del NRC (2012) se cita en la mayoría de los trabajos más recientes sobre prácticas científicas (e.g., Ageitos, Puig y Calvo-Peña 2016;

\footnotetext{
${ }^{4}$ Desde esta visión de aprendizaje comunitario, algunos autores han incorporado al enfoque didáctico basado en prácticas científicas la idea de agencia epistémica (e.g., Miller, Manz, Russ, Stroupe y Berland 2018). Se trata de un concepto importado del ámbito de la epistemología social y, en esencia, se emplea para analizar las formas con las que el alumnado, participante en esas prácticas, contribuye a mejorar el conocimiento de su comunidad de aprendizaje.

${ }^{5}$ El documento se refiere también a prácticas de ingeniería, pero no nos ocuparemos de estas por razones de extensión.
} 
Crujeiras-Pérez y Cambeiro 2018; Crujeiras-Pérez y Jiménez 2018; Jiménez-Liso, MartínezChico, Avraamidou y López-Gay 2019; López, Grimalt-Álvaro y Couso 2018; Mosquera, Puig y Blanco 2018; Muñoz-Campos, Franco-Mariscal y Blanco-López 2020). Todos ellos se ocupan solamente de prácticas científicas epistémicas; lo cual refuerza que, en efecto, la perspectiva de las prácticas científicas que parece desprenderse con más claridad del citado documento es esencialmente epistémica. $\mathrm{O}$, dicho de otra manera, el marco propuesto por el NRC (2012) invita poco a que la vertiente no-epistémica de las prácticas científicas sea promovida en las clases de ciencia.

Tabla 1. Practicas de ciencia establecidas en "A Framework for K-12 Science Education” (NRC 2012, p. 3; traducción propia).

- Formular preguntas

- Desarrollar y usar modelos

- Planificar y llevar a cabo investigaciones

- Analizar e interpretar datos

- Usar pensamiento matemático y computacional

- Elaborar explicaciones científicas

- Participar en la argumentación a partir de pruebas

- Obtener, evaluar y comunicar información

\section{¿Por qué integrar la dimensión no-epistémica en el enfoque basado en prácticas científicas?}

Desde una perspectiva epistemológica, no es sencillo determinar qué son las prácticas científicas. $\mathrm{Si}$ bien, como primera aproximación, podría decirse que estas constituyen un constructo integrado por todo aquello que las científicas y científicos hacen en (y para poder desarrollar) sus investigaciones, abarcando aspectos tanto epistémicos como no-epistémicos (GarcíaCarmona y Acevedo-Díaz 2018).

Pero ¿a qué nos referimos cuando hablamos de epistémico y no-epistémico? De acuerdo con el DRAE, el adjetivo 'epistémico' deriva del término griego episteme, cuya tercera acepción, relacionada con la filosofía, es: "Saber construido metodológica y racionalmente, en oposición a opiniones que carecen de fundamento". De forma que, en la construcción de conocimiento científico, lo epistémico ${ }^{6}$ se asocia a lo racional o cognitivo (Pournari 2008); es decir, al proceso de justificación de ese conocimiento científico en sí (Cardoso 2020). Esto se hace, por ejemplo, mediante el uso de argumentos y explicaciones basadas en pruebas científicas y marcos teóricos de la ciencia. En cambio, lo no-epistémico ${ }^{7}$ se refiere a aquellos factores de naturaleza sociológica, incluyendo aspectos afectivos, contextuales, conductuales, éticos, comunicativos, organizativos, políticos, culturales, etc., que igualmente influyen o intervienen en la construcción de conocimiento científico (Acevedo-Díaz 2006; Carrier 2013; Elliott y McKaughan 2014; Gandolfi 2019; García-Carmona y Acevedo-Díaz 2018; Koster y de Regt 2020; Parker y Winsberg 2018). Y es que, como Knorr-Cetina (1981) señala, “las distinciones entre lo cognitivo y lo social, lo técnico y lo relevante para la carrera, lo científico y lo no científico son constantemente borradas y redibujadas en el laboratorio" (p. 23; traducción propia).

\footnotetext{
${ }^{6}$ No se debe confundir con el adjetivo epistemológico, que está relacionado con la epistemología, y que se define como la rama de la filosofía que estudia el origen, la naturaleza, el alcance y los límites del conocimiento humano (Boyd, Gasper y Trout 1991, p. 777).

${ }^{7}$ De manera general y simplificada, Pournari (2008) identifica lo no-epistémico con los valores no-cognitivos que intervienen en el desarrollo de la ciencia.
} 
La dimensión no-epistémica de las prácticas científicas es concebida como una componente esencial en diversas propuestas de conceptualización sobre la naturaleza de la ciencia. ${ }^{8}$ Por ejemplo, Irzik y Nola (2014) distinguen entre factores cognitivo-epistémicos y social-institucionales para entender cómo se construye la ciencia. ${ }^{9}$ Acogidas a esta misma visión, Dagher y Erduran (2016) añaden a la dimensión social, o no-epistémica, las organizaciones e interacciones sociales, estructuras de poder público y financiación de la ciencia. Con una mirada parecida, Martins (2015) propone el estudio de cuestiones sobre naturaleza de la ciencia distinguiendo dos ejes fundamentales: (i) histórico y sociológico, y (ii) epistemológico. Acevedo-Díaz y GarcíaCarmona (2016) comparten el fondo de estos enfoques y estructuran la comprensión de la naturaleza de la ciencia en cuatro dimensiones: (i) epistemología de la ciencia, (ii) relaciones entre ciencia y tecnología, (iii) sociología interna de la ciencia, y (iv) sociología externa de la ciencia. En esta última propuesta, las dos dimensiones sociológicas integran lo relativo a la perspectiva noepistémica de la actividad científica.

Por consiguiente, puede decirse que los planteamientos para una enseñanza de la ciencia mediante prácticas científicas, que desatienden su dimensión no-epistémica (e.g., NRC 2012), muestran una imagen limitada del quehacer multifacético de las científicas y científicos (García-Carmona y Acevedo-Díaz 2018). Adicionalmente, creemos que las propuestas que, aun reconociendo la importancia de determinadas prácticas sociales en la construcción de la ciencia, solo hablan de prácticas epistémicas, no ayudan a que las primeras sean atendidas en este marco de educación científica. Y ello, por dos motivos fundamentalmente. El primero, por una cuestión semántica: hace años que, en la filosofía de la ciencia (e.g., Pournari 2008) y también en la didáctica de la ciencia (e.g., Acevedo-Díaz 2006), se maneja el adjetivo 'noepistémico' para referirse a aquellos rasgos o aspectos de la ciencia que no encajan en el dominio de lo 'epistémico', tal y como se ha indicado más arriba. Por tanto, resulta difícil entender que las prácticas epistémicas integren aspectos no-epistémicos. El segundo motivo es que no debería confundirse la atención explícita y reflexiva a aspectos sociológicos de la ciencia (García-Carmona, Acevedo-Díaz y Aragón-Méndez 2018) con promover simplemente un aprendizaje de la ciencia basado en la interacción social (Vygotsky 1985). La participación escolar en prácticas no-epistémicas de la ciencia es un asunto más complejo, que requiere de: (i) la transposición didáctica de aspectos comunicativos, éticos, organizativos, normativos, económicos, etc., propios de la actividad científica, (ii) la formulación de objetivos de aprendizaje específicos sobre su naturaleza, justificación/finalidad y puesta en práctica, (iii) el diseño de actividades ad hoc, y (iv) un plan de evaluación apropiado.

Asimismo, la asimilación de las prácticas científicas escolares como prácticas epistémicas, exclusivamente, invita a pensar que los factores no-epistémicos son considerados -en, el mejor de los casos- como accesorios o subordinados a los de tipo epistémico en la construcción de conocimiento científico. Sin embargo, la historia de la ciencia muestra que muchas veces los factores no-epistémicos han tenido un papel tan importante como los epistémicos en el desarrollo de esta (Acevedo-Díaz y García-Carmona 2017). Por citar solo un ejemplo, es bien conocido que la hipótesis de la Deriva Continental, propuesta por el meteorólogo alemán Alfred

\footnotetext{
${ }^{8}$ Los rasgos característicos de las prácticas científicas están siendo considerados aquí como un subconjunto del poliédrico corpus de la naturaleza de la ciencia (García-Carmona y Acevedo-Díaz 2018). En este sentido, además de un enfoque didáctico, las prácticas científicas se están concibiendo como un constructo metacientifico que analizamos para desarrollar el posicionamiento educativo aquí defendido sobre estas.

${ }^{9}$ En los factores cognitivo-epistémicos, Irzik y Nola (2014) incluyen: procesos y objetivos de investigación; valores como la predicción, explicación, consistencia, simplicidad y utilidad; métodos y reglas metodológicas; y conocimiento científico. Mientras que los factores social-institucionales se refieren a: actividades profesionales de las científicas y científicos, ethos científico, certificación y diseminación del conocimiento científico, y valores sociales de la ciencia.
} 
L. Wegener a principios del siglo XX, encontró un fuerte rechazo en la comunidad de geólogos estadounidenses de la época; entre otras razones, por (1) el sentimiento anti-alemán imperante en Estados Unidos (ambos países acababan de enfrentarse en la Primera Guerra Mundial), y (2) los recelos profesionales que causaba en la comunidad de geólogos que la hipótesis fuese propuesta por alguien que no pertenecía a este colectivo científico (García 1996).

En la misma dirección, Elliott y McKaughan (2014) destacan que los factores no-epistémicos influyen en la evaluación de resultados de investigación científica no solo como consideraciones secundarias en situaciones de incertidumbre, sino también como factores que pueden tener incluso prioridad sobre los de tipo epistémico. Parker y Winsberg (2018) utilizan el siguiente ejemplo para ilustrar esa misma idea:
(...) si en una investigación sobre los posibles efectos cancerígenos de una sustancia química, no queda claro si un tumor [que puede estar asociado a esta] debe ser o no clasificado como maligno, el investigador podría decidir clasificarlo como maligno con el pretexto de que sería peor catalogar la sustancia como segura porque terminaría siendo utilizada ampliamente y podría provocar casos adicionales de cáncer (...). La clasificación de ese tumor ambiguo como maligno disminuiría el riesgo de que la sustancia química se llegue a considerar erróneamente segura. ${ }^{10}$ (p. 126; traducción propia y corchetes añadidos)

Además, los factores no-epistémicos son determinantes en los prolegómenos de una investigación científica. Por ejemplo, una de las principales tareas de los equipos científicos al definir una línea de investigación es buscar financiación para desarrollarla. Y es bien sabido que tal financiación estará condicionada, entre otros aspectos, por la coyuntura económica, política y social del momento; el establishment científico dominante; etc. (Carrier 2013; GarcíaCarmona 2020; Kaya, Erduran, Birdthistle y McCormack 2018). Por tanto, como Kreimer (2005) señala, es razonable pensar que dicha práctica, claramente extracientífica, condiciona en gran medida la fabricación de conocimiento cientifico.

La crítica que aquí se expone, relativa al sesgo de ciertos planteamientos de las prácticas científicas hacia factores únicamente epistémicos, ha sido señalada también por otros autores. Uno de ellos es Collins (2015), quien apunta que la desatención a la perspectiva sociológica (o no-epistémica) de las prácticas científicas se traduce en enseñar una versión diluida de la ciencia. Para ilustrarlo, reflexiona sobre la necesidad de tratar en el aula cuestiones como la integridad de los científicos en sus investigaciones al descubrirse, por ejemplo, que las compañías tabacaleras pagan para encontrar resultados que parezcan cuestionar el vínculo entre fumar y padecer cáncer (Collins 2015, p. 1052). Mody (2015) se ubica en una posición similar y describe la actividad científica como desordenada, contradictoria a veces, y más razonable que racional. Por tanto, sugiere que la enseñanza de la ciencia orientada por practicas científicas debería enfatizar que "otras formas" de conocimiento también constituyen una parte esencial de la ciencia (Mody 2015, p. 1030).

Afortunadamente, en la bibliografía internacional sobre el enfoque didáctico basado en prácticas científicas, encontramos algunas propuestas que sí consideran de manera explícita la dimensión no-epistémica. Por ejemplo, Stroupe (2014, 2015) propone cuatro dimensiones en su propuesta para aprender ciencia como práctica, ${ }^{11}$ entre las que distingue: la dimensión epistémica, que define como la base filosófica por la cual esas personas deciden lo que saben y por qué

\footnotetext{
${ }^{10}$ Utilizando criterios estrictamente racionales, o epistémicos, ese tumor debería ser clasificado clínicamente como ambiguo, y la seguridad de la sustancia química como incierta o indeterminada. Se trata de un caso claro de aplicación del principio de precaución, que consiste en tomar medidas ante la sospecha de que determinados productos o tecnologías crean un riesgo grave para la salud pública o el medio ambiente, aunque no se cuente aún con pruebas científicas definitivas de tal riesgo (Andorno, 2008).

${ }^{11}$ Las cuatro dimensiones son: conceptual, social, epistémica y material (Stroupe, 2014, 2015).
} 
están convencidos de que lo saben; y la dimensión social, que la concibe como la manera en que las personas acuerdan normas y rutinas para manejar, desarrollar, criticar y usar ideas (Stroupe 2015, p. 1034).

Duschl (2008) muestra gran afinidad con los planteamientos de Kelly (2008), señalados antes. Sin embargo, en nuestra opinión, es mucho más explícito con la necesidad de distinguir, y al mismo tiempo integrar, aspectos epistémicos y no-epistémicos en el aprendizaje basado en prácticas científicas. Concretamente recomienda que el aprendizaje y evaluación de la ciencia escolar debería atender, de forma integrada y equilibrada, tres dominios (Duschl 2008, p. 277; traducción propia): (a) las estructuras conceptuales y procesos cognitivos usados al razonar científicamente, (b) los marcos epistémicos usados al desarrollar y evaluar conocimiento científico, y (c) los procesos sociales y contextos que determinan cómo el conocimiento es comunicado, representado, argumentado y debatido. La perspectiva no-epistémica la identificamos en el último dominio.

Más recientemente, García-Carmona y Acevedo-Díaz (2018) han hecho una profunda revisión de las principales aportaciones de la historia, la filosofía y la sociología de la ciencia para determinar las prácticas más habituales de las personas que hacen ciencia. La propuesta integra prácticas epistémicas y no-epistémicas; $y$, partiendo de ella, se propone una serie de principios didácticos para desarrollar una enseñanza de la ciencia inspirada en prácticas científicas que, además, favorezca la comprensión de su naturaleza.

\section{¿Qué prácticas no-epistémicas abordar en el enfoque basado en prácticas científicas?}

Una vez que se ha argumentado la necesidad de ampliar el enfoque de aprendizaje basado en prácticas científicas, añadiendo las de índole no-epistémica, cabe preguntarse qué prácticas de este tipo se podrían abordar en la enseñanza de la ciencia. En la bibliografía existen algunas conceptualizaciones interesantes sobre la naturaleza de la ciencia, que incluyen aspectos noepistémicos inherentes a la actividad científica (e.g., Acevedo-Díaz y García-Carmona 2016; Aikenhead y Ryan 1992; Irzik y Nola 2014; Olson 2018). Consideramos que disponer de diferentes propuestas marco es beneficioso porque permitirá al profesorado escoger aquellas prácticas no-epistémicas que le parezcan más factibles o apropiadas, según el nivel educativo y/o contenido de ciencia escolar. No obstante, en la tabla 2 mostramos, a modo de ejemplo, una relación no cerrada de factores no-epistémicos que podrían ser tenidos en cuenta a la hora de determinar posibles prácticas escolares relacionadas con estos. A continuación, ofrecemos algunas orientaciones fundamentadas para hacer este ejercicio de transposición didáctica.

Tabla 2. Relación de algunos factores no-epistémicos que influyen o forman parte de las prácticas cientificas (extraído y adaptado a partir de García-Carmona y Acevedo-Díaz 2018).

- Cooperación y colaboración científica

- Relaciones profesionales y personales en la comunidad científica

- Comunicación científica

- Habilidades retóricas en la persuasión de ideas científicas

- Papel de la comunidad científica en la aceptación de nuevos conocimientos

- Ética en la investigación científica

- Búsqueda de fondos para la investigación 


\section{Práctica no-epistémica asociada a la cooperación y colaboración científica}

Es difícil encontrar un descubrimiento científico reciente que pueda atribuirse a un único científico o científica. El progreso de la ciencia se consigue gracias al trabajo de muchas personas que trabajan, de forma coordinada, cooperativa y colaborativa, en equipos, comunidades y redes científicas. Por tanto, si la ciencia es el resultado de un proceso colectivo, la educación científica debe propiciar que el alumnado se organice en equipos de trabajo para cooperar y colaborar durante los procesos de aprendizaje. Se trata de una perspectiva que, además, sintoniza con los planteamientos de la psicología del aprendizaje enmarcados en el constructivismo social (Vygotsky 1985). Sin embargo, como sucede en la ciencia, ese trabajo colectivo en el aula no está exento de tensiones (Sohr, Gupta y Elby 2018); por tanto, precisa que el alumnado se implique en la elaboración de una serie de reglas o normas para hacerlo funcional y efectivo. Así pues, una práctica no-epistémica esencial en el aprendizaje de la ciencia, al respecto, podría enunciarse como sigue:

- Elaborar estándares de cooperación y colaboración intra- e inter-equipos para participar en indagaciones científicas, intercambiar ideas y planteamientos de indagación, etc.

\section{Prácticas no-epistémicas asociadas a las relaciones profesionales y personales en la comunidad científica}

Relacionado con lo anterior, en el diseño y desarrollo de proyectos de investigación, los equipos de científicas y científicos no solo deben formular hipótesis, establecer objetivos de investigación, describir la metodología, determinar los recursos necesarios, etc. También deben indicar las tareas en las que se estructurará la investigación y cómo será la distribución de estas entre los miembros del equipo. Por tanto, esta idea debería reflejarse en las indagaciones escolares que se propongan.

Las instituciones públicas que financian las investigaciones abogan también por estimular y reconocer la presencia de mujeres en equipos de investigación; de manera que la incorporación de la perspectiva de género se instituye como una cuestión prioritaria y transversal, que tiene que ser considerada en todos los aspectos del proceso investigador. ${ }^{12}$ Con lo cual, ello se erige igualmente como una práctica que ha de promoverse en la constitución y organización de los equipos escolares cuando hacen indagaciones científicas.

Asimismo, el éxito de un proyecto de investigación depende, en buena medida, de las relaciones personales entre los miembros el equipo, del compromiso que adquieren con la investigación y de cómo se superan posibles conflictos durante el desarrollo de esta. En la educación científica, esto ha sido considerado, de algún modo, en estudios sobre la relación entre clima de aula y resultados de aprendizaje de la ciencia (Espadero y Vilches 2018).

Todos estos aspectos pueden promoverse entre el alumnado de ciencia mediante la participación en las prácticas científicas no-epistémicas siguientes:

- Organizar y repartir las distintas tareas de planificación y ejecución de una indagación cientifica escolar, asignando roles a los diferentes miembros del equipo de trabajo.

$\checkmark$ Distribuir las tareas de indagación y los roles de los miembros del equipo, independientemente de su género, y tratando de que exista una proporción equilibrada de ambos géneros.

$\checkmark$ Definir códigos de compromiso personal y de relaciones interpersonales en el equipo para abordar una indagación científica escolar.

\footnotetext{
${ }^{12}$ En España, por ejemplo, esto está establecido en la Disposición adicional decimotercera de la Ley de la Ciencia y la Tecnología (BOE núm. 131, de 2 de junio de 2011).
} 


\section{Prácticas no-epistémicas asociadas a la comunicación científica}

Una de las tareas básicas de las científicas y científicos es difundir los hallazgos de sus investigaciones, así como consultar, interpretar y discutir los de otros colegas. Ello se hace, principalmente, con la elaboración de informes que son publicados en libros, artículos de revistas y actas de reuniones científicas. Consecuentemente, es sensato que la educación científica establezca entre sus objetivos básicos el desarrollo de habilidades para la comunicación de conclusiones derivadas de indagaciones científicas escolares (MEC 2014). Pero ello no pasa solo por la concepción epistémica de comprender -entre otros aspectosque tales conclusiones deben estar fundamentadas a partir de análisis rigurosos de datos y de razonamiento para su interpretación. También requiere saber cómo dar a conocer esas conclusiones, junto con el proceso seguido para llegar a ellas, a fin de que puedan ser entendidas, evaluadas y criticadas por otras personas (Ford 2015; García-Carmona 2012; Reigosa 2012). De hecho, las revistas científicas, las reuniones científicas, etc. establecen normas o estándares de presentación y publicación de trabajos originales de investigación. Con esto en mente, dos prácticas no-epistémicas asociadas a la comunicación científica, que podrían promoverse en clases de ciencia, son las siguientes:

- Determinar conjuntamente estándares de formato (estructura, estética, etc.) y de contenido para la elaboración de informes de indagaciones científicas escolares.

- Establecer mediante consenso los modos y criterios para comunicar y debatir sobre los diferentes resultados o conclusiones de una indagación cientifica.

\section{Prácticas no-epistémicas asociadas a la retórica en la persuasión de ideas científicas}

Ligado a la comunicación científica, el éxito de unas ideas científicas frente a otras no depende solo de la robustez de las pruebas en las que estas se apoyen. Las habilidades de las científicas y científicos para la comunicación de tales ideas también suelen jugar un papel decisivo en su aceptación o rechazo. Por ejemplo, las dificultades de Ignaz Semmelweis para explicar sus ideas científicas sobre la fiebre puerperal, además de otros factores de corte epistémico, fueron determinantes en que estas no fueran reconocidas por la comunidad científica de su época (Acevedo-Díaz y García-Carmona 2017). En general, la importancia de este factor se pone de manifiesto cuando, entre los motivos de la desestimación de un artículo de investigación o de la solicitud formal de un proyecto, se alega que no está "bien escrito".

La redacción de informes científicos por parte del alumnado ya ha sido atendida en la didáctica de la ciencia, y existen trabajos interesantes al respecto (e.g., Domènech 2013; Reigosa 2006). Igualmente han sido analizadas las habilidades comunicativas del alumnado en clase de ciencia (e.g., De Longhi et al. 2012; Reigosa 2012). Por tanto, dos prácticas escolares no-epistémicas que deberían impulsarse, en este sentido, son las siguientes:

- Manejar técnicas básicas (lenguaje verbal, gestual, oratoria, estética, etc.) para la presentación en público de los resultados de una indagación escolar.

$\checkmark$ Participar en debates sobre los hallazgos y conclusiones de una indagación escolar, distinguiendo los argumentos cientificos (basados en pruebas, de causa-efecto, etc.) de los de otra naturaleza (emotivos, éticos, estéticos, de autoridad, basados en creencias extra-cientificas, etc.), aunque valorando también la importancia de estos últimos en la persuasión de ideas o posicionamientos cientificos.

\section{Prácticas no-epistémicas asociadas al papel de la comunidad científica en la aceptación de nuevos conocimientos}

El conocimiento científico es el producto final de la investigación científica y, aunque susceptible de sufrir modificaciones a causa de nuevas pruebas, puede considerarse objetivo 
una vez establecido. Sin embargo, su construcción y proceso de aceptación están impregnados de subjetividad (García-Carmona y Acevedo-Díaz 2018). Por ello, se aplican mecanismos de coevaluación y evaluación externa rigurosos para garantizar la validez y aumentar la objetividad de las conclusiones científicas (evaluación por pares, foros de discusión, intersubjetividad, etc.). Todo esto pone de relieve la importancia de los procesos de evaluación en el desarrollo de la ciencia.

Algunos trabajos ya han tratado la viabilidad y utilidad de tales procesos de coevaluación (o feedback horizontal) entre el alumnado mientras aprende ciencia (e.g., Anker-Hansen y Andrée 2019; Forman y Ford 2014). Así pues, entre las posibles practicas no-epistémicas asociadas con este asunto para el ámbito escolar, pueden impulsarse las siguientes:

- Elaborar, dentro de los equipos de trabajo, criterios para decidir cómo establecer las conclusiones de equipo (mediante consenso, aceptación por mayoría, solicitud de algún control externo - "amigo crítico"-, etc.) en una indagación cientifica escolar.

- Discutir las ventajas e inconvenientes de evaluar trabajos de indagación por pares 'conocidos' vs. pares 'anónimos', y seleccionar el proceso más pertinente en las distintas fases o tareas de una indagación cientifica escolar.

$\checkmark$ Establecer colectivamente criterios (por ejemplo, mediante rúbricas) y procesos de seguimiento (evaluación por pares, elaboración de informes parciales con los progresos y dificultades que se van encontrado, etc.) en el desarrollo y evaluación de una indagación cientifica escolar.

\section{Prácticas no-epistémicas asociadas a la ética en la investigación científica}

A lo largo de la historia de la ciencia se han llevado a cabo numerosas investigaciones científicas éticamente reprobables; especialmente en las realizadas con seres humanos (Medford, Ordóñez, Garzó y Carrazana 2010). De igual forma son rechazables desde un punto de vista ético las investigaciones fraudulentas; a saber, aquellas en las que se inventaron datos, se manipularon resultados o hubo plagio de otros estudios. Por ello, la comunidad científica establece que toda investigación debe estar sometida a unos códigos de buenas prácticas (Puigdomènech 2016), que abarcan desde la selección del objeto de estudio y la planificación de la investigación, hasta la publicación de los resultados y conclusiones. Incluso en determinados campos de la ciencia como, por ejemplo, la biomedicina, existen comités éticos para velar por el desarrollo de buenas prácticas en las investigaciones que se realicen. De modo que, es más que razonable plantear prácticas relacionadas con asuntos éticos en la educación científica desde los niveles básicos. En la bibliografía se encuentran algunas propuestas educativas interesantes para trabajar con estudiantes de distintas edades la ética en la ciencia; como, por ejemplo, la de Jones, McKim y Reiss (2010).

Con todo, algunas prácticas no-epistémicas relacionadas con la ética científica, que estaría bien fomentar en el ámbito escolar, pueden ser las siguientes:

- Establecer colectivamente estándares de comportamiento apropiados en indagaciones cientificas escolares que involucren a seres vivos y otros elementos de espacios naturales, asi como respecto al manejo de material de laboratorio.

- Seleccionar problemas para el desarrollo de indagaciones cientificas escolares que no entrañen conflictos éticos de cualquier tipo.

- Determinar normas éticas a seguir en la recopilación, manejo y análisis de datos empíricos, de información procedente de informes cientificos, fuentes secundarias, etc. 


\section{Práctica no-epistémica asociada a la búsqueda de fondos para la investigación}

Como se ha señalado más arriba, una de las prácticas esenciales de las científicas y científicos es la obtención de fondos para llevar a cabo sus investigaciones (Kreimer 2005). La atención a esto en la educación científica podría traducirse en que el alumnado piense cómo llevar a cabo indagaciones escolares con el menor coste posible, aprovechando los recursos disponibles, elaborando presupuestos sencillos, etc. Esto puede propiciar el desarrollo de capacidades para la planificación y gestión de recursos, así como de concienciación respecto al reciclaje y la reutilización de materiales (García-Carmona 2020). Recientemente, Kaya et al. (2018) han hecho una propuesta interesante para integrar en el currículo de ciencia escolar aspectos relacionados con la economía y el emprendimiento en ciencia.

En consecuencia, dos prácticas no-epistémicas escolares asociadas a todo ello podrían formularse de la siguiente manera:

$\checkmark$ Elaborar presupuestos estimativos del coste de una indagación cientifica escolar para decidir qué opciones pueden resultar económicamente más viables.

- Planear indagaciones cientificas escolares teniendo en cuenta el presupuesto y los recursos disponibles (preferiblemente de bajo coste), incluyendo la posibilidad de reciclar o reutilizar materiales.

\section{Reflexión final}

La posición defendida aquí, en torno al aprendizaje de la ciencia mediante prácticas científicas, no pretende desacreditar o restar importancia a aquellos planteamientos que solo ponen el foco en prácticas epistémicas, ya sea explícita o implícitamente. El propósito es, más bien, ampliar el enfoque añadiendo las prácticas de perfil no-epistémico, que también constituyen una parte esencial de la actividad científica; pero que, sin embargo, suelen recibir mucha menos atención en las clases de ciencia. Consideramos que estas últimas se deberían abordar de manera integrada con las de tipo epistémico, y en proporciones equilibradas, porque así se proyecta una imagen más auténtica y holística de la ciencia (García-Carmona 2020; GarcíaCarmona y Acevedo-Díaz 2018).

Como se acaba de desarrollar, se trataría de articular en una misma actividad ambas perspectivas. Por ejemplo, en relación con la formulación de preguntas en ciencia, si, además de trabajar con el alumnado las características que estas deben tener para que sean científicamente investigables, se le invita a reflexionar y discutir sobre qué preguntas científicas serían más interesantes abordar por motivos personales, sociales, económicos, etc., o viables desde un punto de vista ético, se estarían integrando la vertiente epistémica y la no-epistémica. Similarmente, si en la planificación y ejecución de indagaciones escolares, junto al establecimiento de hipótesis, la determinación de procedimientos para la toma y manejo de datos, etc., se promueve la realización previa de un presupuesto económico para llevarla a cabo, el interés por usar materiales reciclados o reutilizables para los experimentos, la elaboración consensuada de un código deontológico escolar para su desarrollo, etc., se estarían integrando ambas perspectivas también. La clave está, por tanto, en promover ambos dominios de la actividad científica de una manera explícita, mediante la formulación de objetivos de aprendizaje específicos y el diseño de actividades escolares claramente orientadas a ello. Todo esto, sin olvidar que la participación en prácticas científicas, epistémicas y no-epistémicas, requiere que el alumnado ponga en juego procesos cognitivos, habilidades procedimentales, así como conocimientos de ciencia y sobre la naturaleza de tales prácticas (Duschl 2008; GarcíaCarmona y Acevedo-Díaz 2018).

Por otro lado, es posible que, para algunas lectoras y lectores, parte de las prácticas noepistémicas que se acaban de sugerir no supongan realmente una novedad, dentro de la amplia 
bibliografía sobre didáctica de la ciencia. Sin embargo, esta es una circunstancia de la que tampoco escapan las propuestas centradas en prácticas epistémicas, como la que se sugiere en el documento "K-12 Framework" (NRC 2012). Hace ya tiempo que, en la enseñanza de la ciencia, se empezó a promover la atención explícita a prácticas como el planteamiento de preguntas investigables (e.g., Shodell 1995), la formulación de hipótesis (e.g., Wenham 1993), la observación científica (e.g., Haury 2002; Hodson 1986), la medición empírica de magnitudes físicas (e.g., Haury 2003; NRC 1996), la interpretación de datos empíricos (e.g., Kanari y Millar 2004), la modelización (e.g., Buckley, Boulter, Gilbert y Boulter 2000) o la argumentación científica (e.g., Kuhn, 1993; Newton, Driver y Osborne 1999), entre otras. Por tanto, la principal aportación del enfoque basado en prácticas científicas no radica en si las distintas prácticas que se proponen son novedosas o no, sino en cómo se articulan y para qué en clase de ciencia, con vistas a mejorar la competencia científica del alumnado. Efectivamente, lo reseñable es que las diferentes prácticas científicas, aunque deban relacionarse e implementarse de manera integrada en un mismo marco de aprendizaje, tienen entidad didáctica propia. Esto quiere decir que pueden activarse unas u otras, y con la vinculación que se desee, según los intereses y objetivos didácticos de cada situación educativa. Por consiguiente, ello permite huir del planteamiento lineal y secuenciado en pasos ordenados de tareas con el que, a menudo y desafortunadamente ${ }^{13}$, se ha identificado el aprendizaje de la ciencia orientado por el EBI.

\section{Agradecimientos}

Este estudio se enmarca en el proyecto de investigación EDU2017-82505-P, financiado por el Ministerio de Economía, Industria y Competitividad (Gobierno de España).

\section{Referencias}

Abd-El-Khalick, F., Boujaoude, S., Duschl, R., Lederman, N. G., Mamlok-Naaman, R., Hofstein, A., . . . Tuan, H.-L. (2004) Inquiry in science education: International perspectives. Science Education 88 (3), 397-419.

Abell, S. K., Smith, D. C., Volkmann, M. J. (2006) Inquiry in science teacher education. En L. B. Flick y N. G. Lederman (eds.), Scientific inquiry and nature of science (pp. 173-199). Dordrecht: Springer.

Acevedo-Díaz, J. A. (2006) Relevancia de los factores no-epistémicos en la percepción pública de los asuntos tecnocientíficos. Revista Eureka sobre Enseñanza y Divulgación de las Ciencias 3 (3), 370-391.

Acevedo-Díaz, J. A., García-Carmona, A. (2016) «Algo antiguo, algo nuevo, algo prestado». Tendencias sobre la naturaleza de la ciencia en la educación científica. Revista Eureka sobre Enseñanza y Divulgación de las Ciencias 13 (1), 3-19.

Acevedo-Díaz, J. A., García-Carmona, A. (2017) Controversias en la historia de la ciencia y cultura cientifica. Madrid: Los Libros de la Catarata.

Acevedo-Díaz, J. A., García-Carmona, A., Aragón, M. M. (2017) Enseñar y aprender sobre naturaleza de la ciencia mediante el análisis de controversias de historia de la ciencia. Resultados y conclusiones de un proyecto de investigación didáctica. Madrid: OEI.

Ageitos, N., Puig, B., Calvo-Peña, X. (2017) Trabajar genética y enfermedades en secundaria integrando la modelización y la argumentación científica. Revista Eureka sobre Enseñanza y Divulgación de las Ciencias 14 (1), 86-97.

\footnotetext{
${ }^{13}$ Los planteamientos más genuinos para el aprendizaje basado en el EBI (e.g., Abell et al. 2006; Cañal 1999; Harlen 2012) no sugieren seguir exactamente una serie de pasos ordenados y secuenciados, como se propone, por ejemplo, desde la perspectiva del método científico.
} 
Aguilera, D., Martín-Páez, T., Valdivia-Rodríguez, V., Ruiz-Delgado, A., Williams-Pinto, L., Vílchez-González, J. M., Perales-Palacios, F. J. (2018) La enseñanza de las ciencias basada en la indagación. Una revisión sistemática de la producción española. Revista de Educación 381, 259-284.

Aikenhead, G. S., Ryan, A. G. (1992) The development of a new instrument: 'Views on Science-Technology-Society' (VOSTS). Science Education 76 (5), 477-491.

Anderson, R. D. (2002) Reforming science teaching: What research says about inquiry. Journal of Science Teacher Education 13 (1), 1-12.

Andorno, R. (2008). Principio de Precaución. En Diccionario Latinoamericano de Bioética (pp. 345347). Bogotá: UNESCO.

Anker-Hansen, J., Andrée, M. (2019) Using and rejecting peer feedback in the science classroom: A study of students' negotiations on how to use peer feedback when designing experiments. Research in Science \& Technological Education 37 (3), 346-365.

Barrow, L. H. (2006) A brief history of inquiry: From Dewey to standards. Journal of Science Teacher Education 17 (3), 265-278.

Boyd, R., Gasper, P., Trout, J. D. (eds.) (1991) The philosophy of science. Cambridge, MA: MIT Press.

Buckley, B. C., Boulter, C. J., Gilbert, J. K., Boulter, C. J. (2000) Developing models in science education. Dordrecht: Kluwer

Bybee, R. W. (2011) Scientific and engineering practices in K-12 classrooms: Understanding a framework for K-12 science education. Science and Children 49 (4), 10-16.

Cañal, P. (1999) Investigación escolar y estrategias de enseñanza por investigación. Investigación en la Escuela 38, 15-36.

Cañal, P., Porlán, R. (1987) Investigando la realidad próxima: Un modelo didáctico alternativo. Enseñanza de las Ciencias 5 (2), 89-96.

Cardoso, P. C. (2020) De que conhecimento sobre natureza da ciência estamos falando? Ciência \& Educação 26, e20003.

Carrier, M. (2013) Values and objectivity in science: Value-ladenness, pluralism and the epistemic attitude. Science \& Education 22 (10), 2547-2568.

Collins, H. (2015) Can we teach people what science is really like? Science Education 99 (6), 1049-1054.

Crawford, B. A. (2007) Learning to teach science as inquiry in the rough and tumble of practice. Journal of Research in Science Teaching 44 (4), 613-642.

Crujeiras-Pérez, B., Cambeiro, F. (2018) Una experiencia de indagación cooperativa para aprender ciencias en educación secundaria participando en las prácticas científicas. Revista Eureka sobre Enseñanza y Divulgación de las Ciencias 15 (1), 1201.

Crujeiras-Pérez, B., Jiménez, M. P. (2018) Influencia de distintas estrategias de andamiaje para promover la participación del alumnado de secundaria en las prácticas científicas. Enseñanza de las Ciencias 36 (2), 23-42.

Dagher, Z. R., Erduran, S. (2016) Reconceptualizing the nature of science for science education. Why does it matter? Science \& Education 25 (1-2), 147-164. 
De Longhi, A. L., Ferreyra, A., Peme, C., Bermudez, G., Quse, L., Martinez, S., ... Campaner, G. (2012). La interacción comunicativa en clases de ciencias naturales. Un análisis didáctica a través de circuitos discursivos. Revista Eureka sobre Enseñanz̧a y Divulgación de las Ciencias 9 (2), 178-195.

Domènech, J. (2013) Secuencias de apertura experimental y escritura de artículos en el laboratorio: un itinerario de mejora de los trabajos prácticos en el laboratorio. Enseñanza de las Ciencias 31 (3), 249-262.

Duschl, R. (2008) Science education in three-part harmony: Balancing conceptual, epistemic and social learning goals. Review of Research in Education 32, 268-291.

Elliott, K. C., McKaughan, D. J. (2014) Non-epistemic values and the multiple goals of science. Philosophy of Science 81 (1), 1-21.

Espadero, I., Vilches, A. (2018) Clima del aula en la educación científica. Didáctica de las Ciencias Experimentales y Sociales 35, 59-76.

Ford, M. J. (2015) Educational implications of choosing "practice" to describe science in the Next Generation Science Standards. Science Education 99 (6), 1041-1048.

Forman, E. A., Ford, M. J. (2014) Authority and accountability in light of disciplinary practices in science. International Journal of Educational Research 64, 199-210.

Gandolfi, H. E. (2019) In defense of non-epistemic aspects of nature of science: Insights from an intercultural approach to history of science. Cultural Studies of Science Education 14 (3), $557-567$.

García-Carmona, A. (2012) ¿Qué he comprendido? ¿qué sigo sin entender?: Promoviendo la autorreflexión en clase de ciencias. Revista Eureka sobre Enseñanza y Divulgación de las Ciencias 9 (2), 231-240.

García-Carmona, A. (2020) From inquiry-based science education to the approach based on scientific practices. Science \& Education 29 (2), 443-463.

García-Carmona, A., Acevedo-Díaz, J. A. (2018) The nature of scientific practice and science education. Science \& Education 27 (5-6), 435-455.

García-Carmona, A., Acevedo-Díaz, J. A., Aragón-Méndez, M. M. (2018) Comprensión de estudiantes de Secundaria sobre la dimensión sociológica de la naturaleza de la ciencia a partir de la historia de la ciencia. Ápice. Revista de Educación Cientifica 2(2), 43-54.

García, C. M. (1996) El Simposio de la Asociación Americana de Geólogos del Petróleo (Nueva York, 1926) y la Deriva Continental. Llull 19, 91-109.

Garritz, A. (2012) Proyectos educativos recientes basados en la indagación de la química. Educación Química 23 (4), 458-464.

Gil, D., Dumas, A., Caillot, M., Martínez-Torregrosa, J., Ramírez, L. (1988) La resolución de problemas de lápiz y papel como actividad de investigación. Investigación en la Escuela 6, 3-20.

Harlen, W. (2012) Fibonacci Project. Background resources for implementing inquiry in science and mathematics at school. Paris: Fondation La main à la pâte.

Harlen, W. (2013) Assessment \& inquiry-based science education: Issues in policy and practice. Trieste, Italy: IAP. 
Haury, D. L. (2002) Fundamental skills in science: Observation. Columbus, OH: Clearinghouse for Science Mathematics and Environmental Education.

Haury, D. L. (2003) Fundamental skills in science: Measurement. Columbus, OH: Clearinghouse for Science Mathematics and Environmental Education.

Hodson, D. (1986) The nature of scientific observation. School Science Review 68 (242), 17-29.

Hodson, D. (2005) Teaching and learning chemistry in the laboratory: A critical look at the research. Educación Quimica 16 (1), 30-38.

Inter-Academy Partnership (2010) Taking inquiry-based science education into secondary education. A global conference. York, UK: IAP Science Education Program.

Irzik, G., Nola, R. (2014) New directions for nature of science research. En M. R. Matthews (ed.), International handbook of research in history, philosophy and science teaching (pp. 999-1021). Dordrecht: Springer.

Jiménez, M. P. (2012) Las prácticas científicas en la investigación y en la clase de ciencias. En XXV Encuentros de Didáctica de las Ciencias Experimentales (pp. 9-14). Santiago de Compostela: APICE / Universidad de Santiago de Compostela.

Jiménez-Aleixandre, M. P., Crujeiras, B. (2017) Epistemic practices and scientific practices in science education. En K. S. Taber y B. Akpan (eds.), Science Education (pp. 69-80). Rotterdam: Sense.

Jiménez-Liso, M. R., Martínez-Chico, M., Avraamidou, L., López-Gay, R. (2019) Scientific practices in teacher education: The interplay of sense, sensors, and emotions. Research in Science \& Technological Education, 1-24. https://doi.org/10.1080/02635143.2019.1647158

Jones, A., McKim, A., Reiss, M. (eds.) (2010) Ethics in the science and technology classroom: A new approach to teaching and learning. Rotterdam: Sense.

Kanari, Z., Millar, R. (2004) Reasoning from data: How students collect and interpret data in science investigations. Journal of Research in Science Teaching 41 (7), 748-769.

Kaya, S., Erduran, S., Birdthistle, N., McCormack, O. (2018) Looking at the social aspects of nature of science in science education through a new lens. Science \& Education 27 (5-6), 457-478.

Kelly, G. J. (2008) Inquiry, activity and epistemic practice. En R. A. Duschl, y R. E. Grandy (eds.), Teaching scientific inquiry: Recommendations for research and implementation (pp. 99-117). Rotterdam: Sense.

Kelly, G. J., Licona, P. (2018) Epistemic practices and science education. En M. R. Matthews (ed.), History, philosophy and science teaching (pp. 139-165). Dordrecht: Springer.

Kelly, G., McDonald, S., Wickman, P. (2012) Science learning and epistemology. En B. Fraser y K. Tobin (eds.) Second international handbook of science education (pp. 281-291). Dordrecht: Springer.

Kim, M., Tan, A.-L. (2011) Rethinking difficulties of teaching inquiry-based practical work: Stories from elementary pre-service teachers. International Journal of Science Education 33 (4), 465-486.

Knorr-Cetina, K. D. (1981) The manufacture of knowledge: an essay on the constructivist and contextual nature of science. Oxford: Pergamon. 
Koster, E., de Regt, H. W. (2020) Science and values in undergraduate education. Science \& Education 29 (1) 123-143.

Kreimer, P. (2005) Karin Knorr Cetina. La fabricación del conocimiento. Un ensayo sobre el carácter constructivista y contextual de la ciencia. Redes 11 (22), 209-216.

Kuhn, D. (1993) Science as argument: Implications for teaching and learning scientific thinking. Science Education 77 (3), 319-337.

Lemke, J. L. (1998). Teaching all the languages of science: Words, symbols, images, and actions. En Conferencia on science education in Barcelona. Recuperado de http://academic.brooklyn.cuny.edu/education/jlemke/papers/barcelon.htm

López, V., Grimalt-Álvaro, C., Couso, D. (2018) ¿Cómo ayuda la Pizarra Digital Interactiva (PDI) a la hora de promover prácticas de indagación y modelización en el aula de ciencias? Revista Eureka sobre Enseñanza y Divulgación de las Ciencias 15(3), 33020.

Martins, A. F. P. (2015) Natureza da ciência no ensino de ciências: uma proposta baseada em "temas" e "questões". Caderno Brasileiro de Ensino de Física 32 (3), 703-737.

Medford, M., Ordóñez, B., Garzó, R. L., Carrazana, D. (2010) Ética en la ciencia y tecnología: Un enfoque desde la educación médica superior. Revista Médica Electrónica 32 (1), 1-9.

Michaels, S., Shouse, A. W., Schweingruber, H. A. (2008) Ready, set, science! Putting research to work in K-8 science classrooms. Washington, DC: National Academies Press.

Miller, E., Manz, E., Russ, R., Stroupe, D., Berland, L. (2018) Addressing the epistemic elephant in the room: Epistemic agency and the next generation science standards. Journal of Research in Science Teaching 55 (7), 1053-1075.

Ministerio de Educación [MEC] (2014) Real Decreto 126/2014, de 28 de febrero, por el que se establece el currículo básico de la educación primaria. Madrid: Boletín Oficial del Estado.

Minner, D. D., Levy, A. J., Century, J. (2010) Inquiry-based science instruction -What is it and does it matter? Results from a research synthesis years 1984 to 2002. Journal of Research in Science Teaching 47 (4), 474-496.

Mody, C. M. D. (2015) Scientific practice and science education. Science Education 99 (6), $1026-$ 1032.

Mosquera, I., Puig, B., Blanco, P. (2018) Las prácticas científicas en infantil: una aproximación al análisis del currículum y planes de formación del profesorado de Galicia. Enseñanza de las Ciencias 36 (1), 7-23.

Muñoz-Campos V, Franco-Mariscal A. J., Blanco-López A. (2020) Integración de prácticas científicas de argumentación, indagación y modelización en un contexto de la vida diaria. Valoraciones de estudiantes de secundaria. Revista Eureka sobre Enseñanza y Divulgación de las Ciencias 17 (3), 3201.

National Research Council [NRC] (1996) National science education standards. Washington, DC: The National Academies Press.

National Research Council [NRC] (2012) A framework for K-12 science education: Practices, crosscutting concepts, and core ideas. Washington, DC: The National Academies Press.

Newton, P., Driver, R., Osborne, J. (1999) The place of argumentation in the pedagogy of school science. International Journal of Science Education 21 (5), 553-576.

OECD (2019) PISA 2018. Assessment and analytical framework. Paris: OECD Publishing. 
Olson, J. K. (2018) The inclusion of the nature of science in nine recent international science education standards documents. Science \& Education 27 (7-8), 637-660.

Osborne, J. (2014) Teaching scientific practices: Meeting the challenge of change. Journal of Science Teacher Education 25 (2), 177-196.

Parker, W. S., Winsberg, E. (2018) Values and evidence: how models make a difference. European Journal for Philosophy of Science 8 (1), 125-142.

Pournari, M. (2008) The distinction between epistemic and non-epistemic values in the natural sciences. Science \& Education 17 (6), 669-676.

Puigdomènech, (2016) La ética de la ciencia. Mètode 90, 7-11.

Reigosa, C. (2006) Una experiencia de investigación acción acerca de la redacción de informes de laboratorio por alumnos de física y química de primero de bachillerato. Enseñanza de las Ciencias 24 (3), 325-336.

Reigosa, C. (2012) Un estudio de caso sobre la comunicación entre estudiantes en el laboratorio escolar. Revista Electrónica de Enseñanza de las Ciencias 11 (1), 98-119.

Rocard, M., Csermely, P., Jorde, D., Lenzen, D., Walberg, H., Hemmo, V. (2007) Science education now: A renewed pedagogy for the future of Europe. Brussels, Belgium: Directorate General for Research, Science, Economy and Society.

Romero-Ariza, M. (2017) El aprendizaje por indagación: ¿existen suficientes evidencias sobre sus beneficios en la enseñanza de las ciencias? Revista Eureka sobre Enseñanza y Divulgación de las Ciencias 14 (2), 286-299.

Rönnebeck, S., Bernholt, S., Ropohl, M. (2016) Searching for a common ground -A literature review of empirical research on scientific inquiry activities. Studies in Science Education 52 (2), 161-197.

Shodell, M. (1995) The question-driven classroom: Student questions as course curriculum on biology. The American Teacher 57, 278-281.

Sohr, E. R., Gupta, A., Elby, A. (2018) Taking an escape hatch: Managing tension in group discourse. Science Education 102 (5), 883-916.

Stroupe, D. (2014) Examining classroom science practice communities: How teachers and students negotiate epistemic agency and learn science-as-practice. Science Education 98 (3), 487-516.

Stroupe, D. (2015) Describing "science practice" in learning settings. Science Education 99 (6), 1033-1040.

Vygotsky, L. (1985) Pensamiento y Lenguaje. Buenos Aires: Pléyade.

Wenham, M. (1993) The nature and role of hypotheses in school science investigations. International Journal of Science Education 15 (3), 231-240.

Yoon, H. G., Joung, Y. J., Kim, M. (2012) The challenges of science inquiry teaching for preservice teachers in elementary classrooms: Difficulties on and under the scene. Research in Science Education 42(3), 589-608.

Zhang, L. (2016) Is inquiry-based science teaching worth the effort? Some thoughts worth considering. Science \& Education 25 (7-8), 897-915. 